\title{
Práticas educativas parentais e comportamentos de saúde e risco na adolescência: Um modelo preditivo
}

\author{
Parental educational practices and protective behaviors and health risk in adolescents: \\ A predictive model
}

Caroline Guisantes De Salvo Toni[ ${ }^{[a]}$ Edwiges Ferreira de Matos Silvares ${ }^{[b]}$

\footnotetext{
[a] Doutora em Psicologia Clínica pela Universidade de São Paulo (USP), professora colaboradora do Departamento de Psicologia da Universidade Estadual do Centro-Oeste (Unicentro), Irati, PR - Brasil, e-mail: carolineguisantes@yahoo.com.br

${ }^{[b]}$ Professora do Programa de PósGraduação em Psicologia Clínica na Universidade de São Paulo (USP), São Paulo, SP - Brasil, e-mail: efsmsilv@usp.br
}

Recebido: 04/03/2012 Received: 03/04/2012

Aprovado: 18/04/2012 Approved: 04/18/2012

\section{Resumo}

As práticas educativas parentais são altamente relacionadas ao desenvolvimento de comportamentos socialmente competentes, problemas de comportamento e comportamentos relacionados à saúde na adolescência, porém a literatura ainda diverge a respeito da influência direta ou indireta dessas variáveis. Diante disso, o objetivo geral dessa pesquisa foi o de testar a influência direta das práticas educativas maternas e paternas sobre os comportamentos de proteção e risco à saúde na adolescência, bem como as variáveis de competência social e problemas de comportamento. Para atingir tais objetivos, participaram deste estudo 112 adolescentes de 11 a 13 anos, de ambos os sexos. Todos responderam ao Inventário de Estilos Parentais materno e paterno, Inventário de autorrelato para jovens (YSR) e questionário de estilo de vida (HBSC). Os dados foram analisados através de inferência estatística e análises multivariadas. Os resultados demonstraram que as práticas parentais são preditoras de competência social, internalização, externalização e problemas de comportamento, além de comportamentos de risco e proteção à saúde. Em face desses resultados, a pesquisa indica que a influência parental na fase inicial da adolescência ocorre de forma direta, sendo as práticas parentais determinantes de comportamento de saúde e risco.

Palavras-chave: Relações Pais-Filho. Adolescência. Saúde.

\section{Abstract}

The parental educational practices are highly related to the development of socially competent behavior, behavioral problems and behavior related to adolescence health, but the literature still diverge about the direct or indirect influence of these variables. In that context, the overall objective of this research was test the direct influence of maternal and paternal educational practices on the behaviors of protection and risk to the health at adolescence, as well as the social competence variables and behavioral problems. To achieve these goals, participated in this study 112 teenagers 11 to 13 age, in both sexes. They all answered the Maternal and Paternal Parental Style Inventory, the Young Self Relate (YSR) and the questionnaire of lifestyle (HBSC). The data have been analyzed through statistics inferring and multi-varied analysis. The results indicated that

Psicol. Argum. 2013 jul./set., 31 (74), 457-471 
the parental practices are predictor of social competence, internalization, externalization and behavior problems, also to risk behaviors and health protection. With those results, the research indicates that the parental influence in early adolescence occurs directly, and the parenting practices determinants of health behavior and risk.

Keywords: Parent-child relations. Adolescence. Health.

\section{Introduç̃̃o}

A psicologia e as áreas afins da saúde trabalharam, nas últimas décadas, para entender quais são as variáveis determinantes do desenvolvimento do comportamento pró-social e antissocial. 0 assunto é essencial, pois essas variáveis estão ligadas a comportamentos de risco e proteção de crianças e adolescentes.

Vários autores (Cecconello, De Antoni \& Koller, 2003; Gomide, 2001, 2003, 2006; Mondin, 2008; Santos, 2001) afirmam que é no seio familiar que a criança estrutura seus aspectos cognitivos e emocionais. Além disso, dependendo do padrão de interação da família com a criança, tais experiências familiares podem promover ou mesmo prejudicar o desenvolvimento global infantil.

A literatura sobre o assunto (Gomide, 2001, 2003, 2006; Kerr \& Stattin, 2000; Reppold, Pacheco, Bardagi \& Hutz, 2002; Santos, 2001) aponta para a importância das práticas educativas, utilizadas pelos cuidadores durante o desenvolvimento de crianças adolescentes. Elas são compreendidas como as principais influências no comportamento do indivíduo. Nada mais lógico, visto que a família é o primeiro contexto que exerce função reforçadora sobre o comportamento da criança, ou seja, é a família que modela o repertório comportamental infantil inicial. Posteriormente, na adolescência, aquele sujeito será confrontado com valores do grupo de pares, porém os valores reforçados na infância tendem a ser um fator protetor contra o desenvolvimento do comportamento antissocial e de risco (Kumpfer \& Alvarado, 2003; Vuchinich, Bank \& Patterson, 1992).

Nesse sentido, pesquisas (Gomide, 2001, 2003; Lamborn, Mounts, Steinberg \& Dornbusch, 1991; Patterson, Reid \& Dishion, 1992) indicam a relevância da relação entre pais e filhos e, principalmente, das práticas utilizadas por esses pais na educação de seus filhos como estratégias preventivas. Essas estratégias são utilizadas com o objetivo de promover a socialização dos filhos, por isso recebem o nome de práticas educativas parentais (Reppold et al., 2002).

Devido à importância desse tema, as investigações que tratam da interação entre pais e filhos, que enfocam tanto os processos normativos do desenvolvimento quanto os da etiologia de aspectos psicológicos do comportamento de crianças e adolescentes, têm sido uma presença constante nos periódicos científicos (Steinberg, 2000; Steinberg \& Morris, 2001). Na adolescência, em especial, as formas de interação familiar são de extrema significância, haja vista ser esse um dos períodos de desenvolvimento em que o indivíduo se encontra mais vulnerável. Isso acontece em razão das diversas mudanças físicas e psicológicas que o adolescente vivencia (Haggerty, Sherrod, Gamezy \& Rutter, 2000).

De acordo com Williams, Holmbeck e Greenley (2002), a adolescência é marcada por dois pontos de transição de grande relevo: a passagem da infância para o início da adolescência e a transição da adolescência para a vida adulta. Esses dois momentos exigem grandes adaptações e o desenvolvimento de novos repertórios. Dessa forma, não é surpreendente que existam mudanças significativas de um período para o outro, além de frequentes problemas de saúde e transtornos psiquiátricos durante o desenvolvimento da adolescência se comparado à infância (Haggerty et al., 2000). Da mesma forma, a interação dos pais com seus filhos sofre grandes modificações nesse estágio do desenvolvimento.

Na infância, os pais são os principais agentes no ambiente dos filhos. Já na adolescência, a importância dos pares é salientada, e os pais passam a influenciar direta e indiretamente o comportamento do adolescente. Por exemplo, no comportamento de adição, os efeitos diretos das práticas parentais ocorrem por meio da modelagem, do reforçamento social e da qualidade da relação pais-filhos, especialmente ligada ao baixo monitoramento e à falta de consistência (Kandel \& Wu, 1995). Indiretamente, os pais influenciam seus filhos por meio de seus 
próprios comportamentos, como participação em grupos com abuso de substâncias, inconsistência de orientações entre o casal parental, com brigas conjugais, entre outros (Hoffman, 1993).

0 processo pelo qual pais e pares influenciam o comportamento do adolescente parece diferir entre si. A influência parental parece dar-se principalmente pelo fornecimento de modelos e pelas normas proporcionadas nos ambientes. A influência entre pares parece acontecer diretamente por modelagem de papéis, ou seja, o comportamento do adolescente é reforçado ou punido nas suas relações com os pares, no que diz respeito, por exemplo, à assertividade, e também indiretamente, mediante o desenvolvimento de regras dentro do grupo favoráveis a diversos comportamentos de risco, como o uso de drogas, (Hoffman, 1994), ou de saúde, como a prática de exercícios (Williams et al., 2002).

Apesar de a multideterminação dos comportamentos ser amplamente documentada, vários autores divergem sobre qual seria o principal agente de influência na determinação dos comportamentos de saúde e risco na adolescência: pais ou pares. Por exemplo, para Bee (2003), Shaffer (2005), Pinto (2006), Outeiral (2008), o principal determinante na adolescência seriam os pares. Já para autores como Pettit e Bates (1989), Kandel (1996), Oxford, Oxford, Harachi, Catalano e Abbot (2001), Gomide (2003), Fletcher, Steinberg e Willians-Wheeler (2004), são os pais que, a partir de suas práticas educativas, seriam os principais agentes no desenvolvimento de comportamentos de proteção e risco à saúde.

Apesar da divergência dos autores, estudos que avaliaram as práticas parentais e a adolescência constataram que o comportamento de risco do adolescente pode ser minimizado por altos níveis de monitoria e controle de comportamento por parte dos pais (Fletcher et al., 2004).

Na revisão da literatura (Alvarenga, 2001; Catalano \& Abbot, 2001; Cecconello \& Koller, 2000; Cecconello et al., 2003; Löhr, 2001; Martínez \& García, 2007; Mondin, 2008; Oxford, Harachi, Weber, Viezzer \& Brandenburg, 2003; Paiva \& Ronzani, 2009; Patterson et al., 1992; Pettit \& Bates, 1989), constatou-se que problemas envolvendo comportamentos de risco em crianças e adolescentes geralmente estão relacionados com práticas parentais deficitárias: baixa monitoria positiva, negligência, baixo comportamento moral e abuso físico. Porém, essas pesquisas, em geral, avaliam cada prática parental de forma isolada, não levando em consideração o conjunto das práticas utilizadas pelos pais em dadas situações, nem o quanto cada prática, associada a outras, contribui para cada tipo de comportamento. Em outras palavras, existe uma lacuna de pesquisas envolvendo esse aspecto da temática.

$\mathrm{Na}$ adolescência, o contato com fatores de risco e a emissão de comportamentos de risco são comuns, uma vez que, com o passar do tempo, cresce o acesso do adolescente a novos ambientes (Carmo, Cunha \& Costa, 2009). Isso, no entanto, não necessariamente significa algo patológico, pois o desenvolvimento e a cultura em que o jovem se insere naturalmente o levem a ter acesso a novas contingências que podem se configurar como risco e/ou proteção.

A importância dos fatores e comportamentos de risco iniciados na adolescência pode ser ressaltada com base nos resultados dos estudos epidemiológicos, como o de Williams et al. (2002). Nesse estudo, os autores demonstram que as maiores causas de mortes entre adultos são, por exemplo, problemas coronários, câncer, doenças pulmonares, entre outras. Isso ocorre predominantemente devido aos fatores de risco comportamentais como fumar, usar álcool e drogas, manter hábitos alimentares indesejáveis, levar uma vida sedentária etc. Para os autores citados, esses comportamentos estão fartamente documentados como iniciados, em geral, na adolescência e sofrem escaladas no decorrer da vida (Carlini, Galduróz, Noto \& Nappo, 2002; Hawkins, Catalano \& Miller, 1992; Laranjeira, Pinsky, Zaleski \& Caetano, 2007).

Dietas e prática de exercício, apesar de se originarem na infância, se estabelecem de forma permanente na adolescência (Williams et al., 2002), fase em que também os comportamentos sexuais se iniciam (Taquette, Andrade, Vilhena \& Paula, 2005). Do exposto, pode-se concluir sem problemas que a adolescência aparece como principal período de desenvolvimento para programas preventivos.

Apesar de a adolescência se apresentar como período de desenvolvimento altamente correlacionado ao de manutenção de comportamentos de saúde, Carlini-Cotrim, Gazal-Carvalho e Gouveia (2000) afirmam que no Brasil as pesquisas sobre comportamentos de saúde entre jovens ainda são escassas e tendem a se concentrar em questões ligadas à gravidez precoce, ao uso de anticoncepcionais e ao uso de substâncias. Entre estudos recentes sobre o tema, foram encontrados pesquisadores como Malcon, Menezes, Maia, Chatkin e Victoria

Psicol. Argum. 2013 jul./set., 31 (74), 457-471 
(2003), Monteio, Victora e Barros (2004), Carlini et al. (2002), Laranjeira et al. (2007); Farias et al. (2009) e Legnani et al. (2009). Eles têm buscado mapear outros comportamentos de risco e proteção à saúde, porém, em geral, de forma epidemiológica, ou seja, com objetivos diferentes dos que buscam determinantes para os comportamentos. Observase também que esses estudos avaliam o comportamento de risco à saúde de adolescentes, utilizando amostras de várias faixas etárias em conjunto (Farias et al., 2009). Assim, diversos estudos interessantes acabam por ter sua generalidade comprometida, por tratar de forma similar os adolescentes em momentos distintos de desenvolvimento.

A adolescência é um momento propício para intervenções precoces em relação a comportamentos de saúde. Dessa forma, identificar com maior rigor as variáveis que predizem, influenciam e mediam tais comportamentos em momentos distintos da adolescência ainda é um ponto a ser mais bem detalhado dentro da psicologia.

Avaliar e conhecer variáveis preditoras são uma forma de possibilitar intervenção focal em resposta a comportamentos de risco específicos em adolescentes nas diferentes faixas etárias. Eles podem também relacionar, de forma sistemática, as práticas parentais nas diversas faixas etárias da adolescência a variáveis como competência social e problemas de comportamento; podem ainda esclarecer o papel mediador dessas variáveis e responder à pergunta: as práticas parentais podem predizer comportamentos de risco e de saúde entre os adolescentes? E como foco deste estudo, questiona-se: as práticas educativas parentais podem ser consideradas preditoras de comportamentos de proteção/saúde e risco em adolescentes com idades entre 11 e 13 anos, ou, segundo a classificação de Outeiral (2008), na adolescência inicial?

Buscando responder a tais questões, optou-se por utilizar a percepção do adolescente a respeito de seu comportamento e das práticas educativas de seus pais. Esse tipo de dado, atualmente utilizado por diversos pesquisadores (Lamborn, Mounts, Steinberg \& Dornbusch, 1991; Stanton et al., 2000; Steinberg, Lamborn, Darling, Mounts \& Dornbusch, 1994; Totura, MacKinnon-Lewis, Gesten, Gadd, Divine, Dunham \& Kamboukos, 2009), permite mapear os fatores de risco e proteção com base no relato dos próprios adolescentes, ou seja, a população de interesse. Assim, é possível garantir resultados mais próximos da realidade dessa população.

\section{Método}

\section{Participantes}

A amostra foi composta por 112 adolescentes, de ambos os sexos, matriculados em classes do Ensino Fundamental de escolas públicas dos municípios de Curitiba (PR) e São Paulo (SP), com idade entre 11 e 13 anos.

Instrumentos

\section{Inventário de estilos parentais (IEP)}

O IEP (Gomide, 2006) é composto por sete escalas que avaliam duas práticas educativas positivas (monitoria positiva e comportamento moral) e cinco negativas (disciplina relaxada, monitoria negativa, negligência, punição inconsistente e abuso físico); cada uma delas com seis itens em escala Likert de três pontos, sendo $0=$ nunca, 1 = as vezes e 2 = sempre. 0 probando avalia a frequência com que seu pai, mãe ou cuidador utiliza a prática descrita no item e assinala a escala. 0 índice de cada prática educativa é calculado com base no somatório simples dos seis itens da escala; o índice de Estilo Parental é encontrado somando-se os pontos obtidos nas escalas das práticas positiva que são subtraídos do somatório dos pontos das cinco escalas das práticas negativas. 0 índice quando negativo informa a prevalência de práticas educativas negativas e quando positivo, a prevalência de práticas positivas no processo educacional.

\section{Questionário helth behavior in school-age children (HBSC/OMS)}

O HBSC/OMS (Currie, Samdal, Boyce \& Smith, 2001), elaborado pela Organização Mundial de Saúde (OMS) e adaptado para diversos idiomas, é composto por 118 questões, as quais se dividem nos seguintes tópicos: 1 - informações sociodemográficas; 2 - hábitos alimentares e de higiene; 3 - imagem corporal; 4 - práticas de atividades físicas e uso de tempo livre; 5 - uso de substâncias; 6 - violência; 7 ambiente familiar; 8 - relações de amizade e grupo de pares; 9 - ambiente escolar; 10 - saúde positiva (aqui entendida como bem-estar geral, satisfação com a vida e comportamentos saudáveis); e 11 comportamentos sexuais. 0 número de questões 
em cada tópico é irregular e o adolescente deve responder a cada item de acordo com uma escala de frequência que varia de item para item.

\section{Questionário de auto avaliação para jovens (YSR)}

0 YSR (Achenbach, 1991), respondido pelos adolescentes em relação aos seus próprios comportamentos, permite agrupar os resultados em dois âmbitos: 1 - competência social; e 2 - problemas de comportamento. A competência social é avaliada a partir das escalas individuais de atividade e sociabilidade, e a soma dos escores brutos das duas escalas fornece o escore final de competência social. Os problemas de comportamento, por sua vez, são derivados das escalas de internalização e externalização. A escala de internalização se subdivide nas escalas individuais de retraimento, queixas somáticas e ansiedade/depressão; já a escala de externalização se subdivide em comportamento de quebrar regras e comportamento agressivo.

Há ainda as escalas individuais de problemas com o contato social, problemas com o pensamento e problemas de atenção. A soma da pontuação bruta obtida nas escalas individuais leva ao escore da Escala Total de Problemas de Comportamento. Os escores brutos são convertidos em escore T, ou seja, escore padronizado. Esse escore padronizado é utilizado, tendo em vista que cada uma das escalas conta com um número específico de itens e o escore $\mathrm{T}$ uniformiza os resultados, permitindo a comparação dos escores obtidos pelo adolescente em todas as escalas do instrumento.

A partir da padronização norte-america são considerados escores clínicos aqueles acima de 70; na faixa borderline se encontraram aqueles com escore entre 67 e 70; e normal, os abaixo de 67. Para o escore das escalas de problemas de comportamento, a faixa borderline se situa entre 60 e 63 pontos; normal, abaixo de 60; e clínico, acima de 63. Para a escala de competência social é considerado normal escore acima de 40, sendo considerados clínicos escores abaixo de 37 e a limítrofe, entre 37 e 40.

\section{Termo de Consentimento Livre e Esclarecido}

Os responsáveis legais pelos adolescentes foram esclarecidos sobre: objetivos do projeto, procedimentos da pesquisa, garantia de direito de confidencialidade e retirada de consentimento de participação na pesquisa no momento em que achassem conveniente através de comunicado da coordenação da escola. Os responsáveis que concordaram com a participação dos menores sob sua tutela assinaram um termo de consentimento livre e esclarecido sobre esses aspectos enviado para a casa por intermédio dos adolescentes e recolhido pela coordenação da escola antes da coleta de dados.

\section{Procedimentos}

\section{Adaptação do Questionário HBSC para o português do Brasil}

O questionário HBSC foi originalmente confeccionado em inglês e português de Portugal e foi adaptado para o português do Brasil pela pesquisadora e posteriormente avaliado por três professores de língua portuguesa, que fizeram os ajustes necessários de ortografia e semântica. Essa versão adaptada foi pré-testada com adolescentes da faixa etária da amostra, mostrando-se adequado.

Após o contato com as escolas públicas, os TCLE foram enviados aos responsáveis pelos adolescentes. Aqueles que aceitaram participar da pesquisa responderam aos questionários na própria escola. A aplicação deu-se de forma coletiva nas salas de aula das escolas participantes, durante o período das aulas.

No início de cada aplicação foi apresentado aos alunos o objetivo da pesquisa, bem como foi garantida a confidencialidade dos dados coletados e reiterada a participação voluntária na pesquisa.

\section{Análise dos dados}

\section{Correção dos Instrumentos e formação da base de dados}

Para a correção do instrumento YSR, utilizou-se o software ADM, programa específico para correção do instrumento. Utilizaram-se na base de dados da pesquisa os escores $\mathrm{T}$ referentes a cada subescala $\mathrm{e}$ escalas do instrumento, visto que esse padroniza os resultados, não havendo interferência do número de itens das escalas. O IEP foi corrigido de forma manual. Utilizaram-se na base de dados os valores brutos referentes a cada prática educativa e ao Índice de estilo parental final do pai e da mãe. O HBSC não possui correção formal, visto que esse é avaliado a partir das respostas a cada questão e/ou ao somatório de questões relativas a um mesmo tópico ou subtópico (dentro de

Psicol. Argum. 2013 jul./set., 31 (74), 457-471 
um mesmo tópico observam-se itens relativos a esferas de comportamento distintas).

A fim de se avaliar o comportamento de saúde/proteção e risco entre adolescentes a partir do HBSC, buscou-se formar escalas com os comportamentos de um mesmo tópico. As escalas formadas a partir de conjuntos de itens do HBSC que versavam sobre comportamentos de proteção/saúde são: 1 satisfação com a vida; 2 - realização de atividades físicas; 3 - sentimentos positivos em relação à escola; 4 - expressão de sentimentos e ideias. Já as escalas de risco incluem: 1 - bullying-agressor; 2 - bullying-vítima; 3 - sintomas somáticos; 4 - uso de drogas; 5 - uso de tabaco; 6 - uso de álcool; 7 - comportamento sexual; 8 - horas em frente a TV/computador.

Para avaliar se as escalas formadas apresentavam boa correlação entre seus itens (validade de constructo), ou seja, se os itens agrupados se relacionam a um mesmo constructo (unidimensionalidade), procedeu-se à análise fatorial de cada uma das escalas formadas, com avaliação de alpha de Cronbach. Como todas apresentaram adequada validade de constructo (Salvo, 2010), utilizou-se a média dessas escalas como representativas dos comportamentos de proteção/saúde e risco.

\section{Análises estatísticas}

Utilizaram-se análise multivariada, modelos de regressão múltipla (stepwise) para análises de predição tomando os escores do IEP como variáveis independentes, e escalas do YSR e escalas do HBSC como variáveis independentes. Para tanto, foi utilizado o software estatístico SPSS 12 for Windows (SPSS, Inc., 1994).

\section{Análise dos resultados}

Buscando maximizar a compreensão dos dados, são apresentados nesta seção de forma conjunta os resultados e a discussão da pesquisa.

Inicialmente avaliaram-se as respostas dos participantes nos três instrumentos, comparando-os quanto à cidade de moradia e sexo. Como não houve diferenças significativas (Salvo, 2010), optou-se por conduzir as análises da amostra em conjunto, sem diferenciação da amostra em função de sexo ou cidade de moradia.
Quando se busca compreender o efeito de uma variável sobre outra, é necessária a aplicação de análise multivariada. Grande parte das pesquisas delineadas para examinar o efeito exercido por duas ou mais variáveis independentes sobre uma variável dependente utiliza a análise de regressão múltipla. A Regressão Múltipla (RM) é definida por Hair, Anderson, Tatham e Black (2005) como um conjunto de técnicas estatísticas que possibilita a avaliação do relacionamento de uma variável dependente com diversas variáveis independentes.

Em face do objetivo deste trabalho - avaliar em que grau as práticas educativas parentais poderiam predizer o comportamento dos adolescentes -, neste estudo utilizou-se o método de regressão stepwise; em outras palavras, de forma aleatória todas as práticas educativas (variáveis independentes) do IEP foram testadas como preditoras das escalas de comportamento do YSR (variáveis dependentes) e para as escalas de comportamento de risco e proteção. Dessa forma, todas as práticas encontradas como preditoras das subescalas do YSR e dos comportamentos de risco e proteção estavam relacionadas a relações significativas entre elas.

Partindo dos resultados do IEP e do YSR, buscou-se inicialmente verificar o quanto as práticas parentais maternas e paternas poderiam ser preditoras das variáveis analisadas pelo YSR: problemas de internalização, problemas de externalização, problemas gerais de comportamento e competência social. Os resultados podem ser observados na Tabela 1 .

Os resultados apontaram para coeficientes de determinação $\left(R^{2}\right)$ variando de 0,20 a 0,26 , ou seja, as práticas educativas do IEP explicaram de 20 a $26 \%$ da variância total das escalas do YSR, valores bastante significativos, em se tratando de comportamento multideterminado. Enquanto os coeficientes de regressão explicam a variância total, os coeficientes de regressão padronizados indicam a proporção em que as mudanças nas unidades de desvio padrão da variável preditiva afetam as mudanças no desvio padrão da variável dependente. Como salientam Floyd, Evans e McGrew (2003), coeficientes de regressão padronizados variando de 0,10 a 0,29 exprimem relações moderadas entre as variáveis, enquanto coeficientes acima de 0,30 representam fortes relações entre as variáveis da equação. Nos modelos de predição formulados na presente pesquisa, encontraram-se valores negativos de coeficiente de regressão padronizado variando entre 
Tabela 1 - Variáveis do IEP preditoras das escalas do YSR para faixa etária 1 (11 a 13 anos)

\begin{tabular}{|c|c|c|c|c|c|c|c|}
\hline Variável dependente (YSR) & $\mathbf{R} 2$ & $\mathbf{F}$ & Gl & $\mathbf{p}$ & Variáveis Preditoras (IEP) & $\begin{array}{l}\text { Beta padroni- } \\
\text { zado }\end{array}$ & $\mathbf{p}$ \\
\hline \multirow{4}{*}{ Competência social } & \multirow{4}{*}{0,26} & \multirow{4}{*}{9,28} & \multirow{4}{*}{34} & \multirow{4}{*}{0,000} & Negligência mãe & $-0,35$ & 0,001 \\
\hline & & & & & Comportamento moral mãe & 0,22 & 0,01 \\
\hline & & & & & Negligência pai & $-0,24$ & 0,01 \\
\hline & & & & & Abuso físico mãe & $-0,20$ & 0,02 \\
\hline \multirow{4}{*}{ Escala de internalização } & \multirow{4}{*}{0,24} & \multirow{4}{*}{8,34} & \multirow{4}{*}{34} & \multirow{4}{*}{0,000} & Disciplina relaxada mãe & 0,18 & 0,04 \\
\hline & & & & & Abuso físico pai & 0,19 & 0,03 \\
\hline & & & & & Monitoria negativa mãe & 0,24 & 0,008 \\
\hline & & & & & Monitoria positiva pai & $-0,23$ & 0,01 \\
\hline \multirow{3}{*}{ Escala de externalização } & \multirow{3}{*}{0,21} & \multirow{3}{*}{9,79} & \multirow{3}{*}{53} & \multirow{3}{*}{0,000} & Negligência pai & 0,24 & 0,01 \\
\hline & & & & & Monitoria negativa mãe & 0,28 & 0,002 \\
\hline & & & & & Comportamento moral pai & $-0,23$ & 0,02 \\
\hline \multirow{3}{*}{$\begin{array}{l}\text { Escala total de problemas de com- } \\
\text { portamento }\end{array}$} & \multirow{3}{*}{0,20} & \multirow{3}{*}{9,50} & \multirow{3}{*}{33} & \multirow{3}{*}{0,000} & Monitoria positiva pai & $-0,33$ & 0,000 \\
\hline & & & & & Monitoria negativa mãe & 0,19 & 0,02 \\
\hline & & & & & Abuso físico pai & 0,19 & 0,03 \\
\hline
\end{tabular}

Fonte: Dados da pesquisa.

$-0,20$ e - 0,35 , enquanto os valores positivos variaram de 0,18 a 0,28 , indicando valores altamente representativos. Os coeficientes de regressão padronizados negativos referem a influência em sentido inverso da variável independente na dependente. Em suma, os resultados sugerem que determinadas práticas educativas podem predizer, significativamente, o comportamento do adolescente.

A partir da Tabela 1, podem-se observar os diversos modelos preditivos encontrados. No primeiro modelo, que buscou avaliar as variáveis preditoras da competência social dos adolescentes, observa-se que o comportamento moral materno, aliado a baixas taxas de negligência materna e paterna e de baixo abuso físico materno, prediz 26\% da competência social, ou seja, essas práticas parentais explicam mais de $1 / 4$ do comportamento socialmente competente dos filhos. A prática educativa comportamento moral se relaciona, entre outras coisas, a modelos parentais socialmente aceitáveis e habilidades de interação. Também contempla as práticas utilizadas pelos pais para o desenvolvimento do próprio comportamento moral, por exemplo, a partir da reparação de danos quando de um comportamento antissocial (Gomide, 2010).

Assim, valores como empatia, generosidade, entre outros, fundamentais na competência social, surgem também das interações com essas práticas parentais. Conte (1997) descreve alguns fatores protetores na adolescência como suporte parental (afeto, valorização do indivíduo etc.), encorajamento do desenvolvimento da competência social e uso de métodos disciplinares não coercitivos. No modelo encontrado, baixos índices de negligência e abuso físico também foram preditores da competência social. Esses resultados estão de acordo com o esperado, tendo em vista que quando negligência e abuso físico ocorrem em alta intensidade, estão correlacionadas tanto ao desenvolvimento de comportamento antissocial quanto a dificuldades de interação (Crowley, Mikulich, Ehlers, Shannon \& Whitmore, 2003; Dodge, Pettit \& Battes, 1994; Salvo, Silvares \& Toni, 2005).

O segundo modelo encontrado, que pode ser observado na Tabela 1 , diz respeito à escala de internalização: disciplina relaxada e monitoria negativa materna, aliadas a abuso físico do pai e ausência de monitoria paterna explicaram $24 \%$ da internalização dos adolescentes de 11 a 13 anos. A literatura descreve como as práticas parentais inconsistentes aliadas a alta fiscalização/controle contribuem para o isolamento do adolescente, na tentativa de proteger-se de um ambiente inconstante e aversivo (Gomide, 2006; Sabbag, 2003; Sapienza \& Pedromônico, 2005), podendo inclusive gerar transtornos de ansiedade (Fletcher et al., 2004); somado a esse contexto, o abuso físico paterno, em geral associado a punições mais severas, colabora para que o adolescente desenvolva comportamentos internalizantes, ou seja, timidez,

Psicol. Argum. 2013 jul./set., 31 (74), 457-471 
dificuldades de comunicação, ansiedade, depressão (Sá, Bordin \& Martin, Paula, 2010; Simons, Wu, Lin, Gordon \& Conger, 2000). As punições constantes e não contingentes produzem um padrão de comportamento retraído e de pouca variabilidade (Hunziker \& Samelo, 2011). Coerção, apresentada geralmente sob a forma de autoritarismo, punições físicas e verbais, disciplina severa, entre outros, geram subprodutos muito negativos para aqueles que a vivenciam, tais como "violência, agressão, depressão, inflexibilidade emocional e intelectual" (Sidman, 1995, p. 248). Os efeitos colaterais da coerção impedem o adequado relacionamento do individuo com a sociedade, desenvolvendo um padrão de esquiva constante, na tentativa de não ser mais punido (Lewinsohn, Rohde, Seeley, Klein \& Gotlib, 2000; Sidman, 1995). Essa esquiva justamente pode desenvolver-se sob a forma dos transtornos de internalização (Masten \& Garmezy, 1985).

Ao contrário da coerção, a monitoria positiva é uma das práticas parentais que mais produtos desejáveis traz (Maccoby \& Martin, 1983; Stattin \& Kerr, 2000). Para Gomide (2004, 2006, 2010), as contingências de reforço positivo são uma fonte fundamental para o controle de comportamentos, visto que fortalecem comportamentos desejados ao invés de interromper comportamentos indesejados. Assim, quando a monitoria positiva está ausente, como no modelo anteriormente apresentado, parece se fechar a contingência para a internalização. Pesquisas (Camacho \& Matos, 2007; Salvador, 2007) verificaram que os jovens a quem os pais dão autonomia e carinho tendem a apresentar melhor rendimento acadêmico, bem como menor predisposição para apresentar transtornos de ansiedade. Os jovens que recebem excessiva proteção (fiscalização) por parte dos pais têm tendência a desenvolver sintomas de ansiedade.

0 terceiro modelo encontrado diz respeito aos preditores da escala de externalização; novamente a negligência paterna, monitoria negativa materna e ausência de comportamento moral paterno predizem $21 \%$ da escala. Os comportamentos que compõem a escala de externalização dizem respeito a quebrar regras, emitir respostas agressivas, entre outras. Nurco e Lerner (1996) descrevem a importância do modelo paterno na proteção contra comportamentos de externalização e de adição. Segundo os autores, é a figura paterna quem garante um ambiente com elevado nível de modelo moral que previne os distúrbios de comportamento.
Barber e Harmon (2002) descrevem como o controle psicológico pode também caracterizar-se como negligência. Para os autores, pais podem utilizar de distanciamento, privação de afeto e indução de culpa na tentativa de controlar seus filhos. Essas respostas são as encontradas nas práticas parentais aqui denominadas de negligência e monitoria negativa. As práticas de privação de afeto também podem ser consideradas técnicas de controle aversivo, pois o individuo se comporta para evitar que algo aconteça. Nesse sentido, o comportamento externalizante pode ocorrer na tentativa de contracontrolar (Sidman, 1995; Patterson et al., 1992). Também, conforme relatos tanto de filhos como de pais, as práticas mais ligadas a valores e regras tendem a ser emitidas pela figura paterna (Weber, Viezzer \& Brandenburg, 2003). No presente modelo, a ausência do comportamento moral paterno também foi uma das variáveis preditoras de externalização.

0 quarto modelo apresentado na Tabela 1 refere-se à escala total de problemas de comportamento, ou seja, ao conjunto das escalas de internalização, externalização, além de problemas com o contato social, problemas de atenção e de pensamento. Para essa escala, foram preditores a ausência de monitoria positiva paterna, a monitoria negativa materna e abuso físico paterno, três práticas já associadas a problemas de comportamento nos modelos anteriores. Vale destacar que a ausência de monitoria positiva paterna teve um alto índice de Beta padronizado $(-0,33)$, ou seja, a ausência de monitoria positiva paterna foi altamente relacionada aos problemas de comportamento. Pesquisas (Kerr \& Stattin, 2000; Matthews, Woodall, Kenyon \& Jacob, 1996; Menegatti, 2002; Muris, Meesters, Morren \& Moorman, 2004; Novak \& Peláez, 2004; Stattin \& Kerr, 2000) sugerem que cuidado, atenção e afeição formam um conjunto de reforçadores que devem ser mantidos e disponibilizados sempre, mesmo quando o filho não corresponde às expectativas dos pais, sendo essa prática protetiva. Para Severe (2000) e Weber (2007), quando o filho se sente amado pelos seus pais, torna-se muito mais fácil discipliná-los.

Buscando-se avaliar se as práticas educativas parentais poderiam ser preditoras dos comportamentos de proteção e risco, procedeu-se à análise de regressão múltipla; os resultados estão apresentados na Tabela 2. 
Tabela 2 - Variáveis do IEP preditoras das escalas de proteção e comportamento de risco para faixa etária 1 (11 a 13 anos)

\begin{tabular}{|c|c|c|c|c|c|c|c|}
\hline Variável dependente (YSR) & $\mathbf{R 2}$ & $\mathbf{F}$ & Gl & $\mathbf{p}$ & Variáveis preditoras (IEP) & Beta padronizado & $\mathbf{p}$ \\
\hline \multirow{2}{*}{ Comportamento assertivo } & \multirow{2}{*}{0,10} & \multirow{2}{*}{6,46} & \multirow{2}{*}{22} & \multirow{2}{*}{0,002} & Disciplina relaxada mãe & $-0,25$ & 0,01 \\
\hline & & & & & Comportamento moral mãe & 0,18 & 0,04 \\
\hline \multirow{2}{*}{ Satisfação com a vida } & \multirow{2}{*}{0,14} & \multirow{2}{*}{9,2} & \multirow{2}{*}{22} & \multirow{2}{*}{0,000} & Negligência mãe & $-0,24$ & 0,009 \\
\hline & & & & & Abuso físico pai & $-0,24$ & 0,01 \\
\hline \multirow{2}{*}{ Envolvimento em lutas } & \multirow{2}{*}{0,20} & \multirow{2}{*}{13,57} & \multirow{2}{*}{22} & \multirow{2}{*}{0,000} & Disciplina relaxada pai & 0,29 & 0,001 \\
\hline & & & & & Comportamento moral pai & $-0,28$ & 0,002 \\
\hline \multirow{3}{*}{ Bullying- gressor } & \multirow{3}{*}{0,16} & \multirow{3}{*}{6,73} & \multirow{3}{*}{33} & \multirow{3}{*}{0,000} & Disciplina relaxada mãe & 0,17 & 0,000 \\
\hline & & & & & Monitoria positiva pai & $-0,35$ & 0,005 \\
\hline & & & & & Monitoria negativa mãe & 0,27 & 0,02 \\
\hline \multirow{4}{*}{ Tabaco } & \multirow{4}{*}{00,22} & \multirow{4}{*}{77,46} & \multirow{4}{*}{34} & \multirow{4}{*}{0,000} & Monitoria positiva pai & $-0,36$ & 0,000 \\
\hline & & & & & Montoria negativa mãe & 0,21 & 0,01 \\
\hline & & & & & Comportamento moral mãe & $-0,20$ & 0,02 \\
\hline & & & & & Abuso físico pai & $-0,19$ & 0,03 \\
\hline \multirow{3}{*}{ Pares } & \multirow{3}{*}{00,13} & \multirow{3}{*}{55,58} & \multirow{3}{*}{53} & \multirow{3}{*}{0,001} & Disciplina relaxada mãe & 0,20 & 0,04 \\
\hline & & & & & Monitoria positiva mãe & 0,27 & 0,007 \\
\hline & & & & & Punição inconsistente mãe & 0,24 & 0,000 \\
\hline \multirow{2}{*}{ Álcool } & \multirow{2}{*}{00,31} & \multirow{2}{*}{224,21} & \multirow{2}{*}{22} & \multirow{2}{*}{0,000} & Comportamento moral pai & $-0,42$ & 0,000 \\
\hline & & & & & Monitoria positiva pai & $-0,22$ & 0,01 \\
\hline
\end{tabular}

Fonte: Dados da pesquisa.

As escalas sentimentos positivos em relação à escola, sintomas somáticos e tempo gasto com TV/ computador apresentaram coeficientes de determinação baixos $\left(R^{2}<0,10\right)$, ou seja, as práticas parentais foram responsáveis por menos de $10 \%$ do total de variação explicada pela equação de regressão múltipla e a variação total da variável dependente. Floyd et al. (2003) descrevem que coeficientes de determinação inferiores a 0,10 apresentam fracas relações e, por isso, sugerem a retirada de tais relações em modelos de predição. Assim, os resultados dessas escalas de comportamento foram excluídos da presente análise. Já as escalas drogas, comportamento sexual e andar com armas, porque apresentaram variação muito pequena na presente amostra (todos os participantes tiveram pontuação mínima nas duas primeiras escalas e na última, apenas quatro relataram vida sexual), não permitiram testar o modelo de regressão.

Dentre os modelos apresentados na Tabela 2, a escala de proteção comportamento assertivo foi predita em apenas $10 \%$ pelas práticas disciplina relaxada e comportamento moral materno; quando a mãe apresenta padrão consistente, mantendo as regras no lar, bem como um padrão de comportamento moral adequado, os filhos tendem a apresentar comportamento assertivo. Essas práticas são descritas na literatura como essenciais para o desenvolvimento da assertividade. Bronstein, Fox e Kamon (2007), buscando avaliar preditores de "coragem moral", ou seja, falar ou agir quando testemunhavam ou vivenciavam injustiça ou algum dano, observaram que aqueles adolescentes que aos 11 anos tinham pais com práticas responsivas e de suporte no tangente a comportamentos morais, foram preditoras de "coragem moral" nas meninas quando estas estavam com 18 anos; nos meninos, os autores encontraram correlações significativas. Apesar de as práticas parentais explicarem apenas $10 \%$ do comportamento assertivo dos filhos, toma-se tais relações como importantes, considerando-se a associação protetiva do comportamento assertivo em relação aos comportamentos de risco e a importância dos pais nesse desenvolvimento (Dias, Matos \& Gonçalves, 2007).

0 segundo modelo apresentado na Tabela 2 refere-se aos preditores da satisfação com a vida; ausência de negligência materna e de abuso físico paterno responde por $14 \%$ da satisfação com a

Psicol. Argum. 2013 jul./set., 31 (74), 457-471 
vida entre adolescentes de 11 a 13 anos. Autores como Dishion e McMahon (1998), Sidman (1995) e Gomide $(2004,2006,2010)$ descrevem que famílias com baixo controle aversivo (punições e privações) tendem a ter filhos mais felizes e competentes, visto que o ambiente familiar favorece o uso de reforçamento positivo. As consequências reforçadoras estabelecem um bom nível de satisfação com os eventos de vida.

Para os comportamentos de risco, foram encontrados como preditores do envolvimento em lutas as práticas paternas disciplina relaxada e ausência de comportamento moral. Ambas confirmam os resultados encontrados por Nurco e Lerner (1996) de que o comportamento paterno é o principal determinante dos comportamentos dos jovens no inicio da adolescência. 0 comportamento de bullying-agressor teve como variáveis preditoras a disciplina relaxada materna, a ausência de monitoria positiva paterna, e a monitoria negativa materna. Segundo Olweus (1980), que descreveu alguns fatores familiares relacionados ao comportamento agressivo, o negativismo materno e a permissividade materna para agressão seriam algumas dessas variáveis. Para Flouri e Buchanan (2003), além das variáveis já descritas por Olweus (1980), o baixo envolvimento do pai e da mãe também estariam relacionados ao comportamento de bullying. 0 modelo encontrado apresenta similaridades com os estudos descritos: a disciplina relaxada em geral ocorre porque a mãe cede nas regras colocadas ante o comportamento agressivo do filho. Também a ausência de monitoria positiva está relacionada ao baixo envolvimento, ao passo que a monitoria negativa materna é justamente caracterizada por um padrão negativo de relações, ou seja, baseada no controle aversivo.

Os modelos de predição de uso de tabaco e uso de álcool guardam similaridades. Em ambos, a ausência de monitoria positiva paterna e de comportamento moral colaborou com o modelo. Para o uso de tabaco, a monitoria negativa materna e o abuso físico do pai ainda contribuíram com o modelo. Cohen, Richardson e LaBree (1994) relacionaram a monitoria parental como fator protetor ao uso de álcool e tabaco; Weber e Gomide (2004) encontraram relações entre o comportamento moral e menores índices de problemas de comportamento, entre eles o uso de álcool e tabaco. 0 abuso físico também é largamente relatado como variável relacionada ao uso de tabaco e álcool. Pesquisa de revisão bibliográfica de Guimarães, Hochgraf, Brasiliano e Ingberman (2009) destaca que a ausência de valores morais na família; e a ausência de um padrão claro de posicionamento contra o consumo de substâncias colabora para o uso de álcool, tabaco e drogas. Também o abuso físico tende a enfraquecer as relações familiares, abrindo espaço para menor monitoramento e aumento das relações baseadas no controle aversivo (Cecconello et al., 2003).

Para a escala pares, as práticas maternas disciplina relaxada, monitoria positiva e punição inconsistente são preditoras e correlacionadas de forma positiva. Ressalta-se que essa escala é formada pelos itens 'sair à noite com amigos' e 'permanecer com amigos fora do horário escolar'. Para adolescentes de 11 a 13 anos, esses comportamentos podem decorrer de: disciplina relaxada, associada a baixa manutenção de regras; monitoria positiva, ou seja, os pais conhecem os amigos e sabem onde os filhos se encontram; tentativa de fuga de controle aversivo, caracterizado pela punição inconsistente. 0 fato de as três práticas estarem relacionadas à escala pares pode indicar um misto dessas práticas, onde o comportamento em relação aos pares pode variar em sua determinação, no que diz respeito às práticas parentais.

Buscando-se avaliar se a inclusão das variáveis pessoais internalização, externalização, problemas de comportamento e competência social como variáveis independentes, junto às práticas educativas parentais, aumentaria o grau de predição dos comportamentos de saúde e risco para adolescentes de 11 a 13 anos, procedeu-se a novas análises de regressão múltipla (stepwise). Para todos os comportamentos de saúde e risco, as variáveis pessoais foram excluídas do modelo, indicando que para adolescentes de 11 a 13 anos, entre as variáveis abordadas neste estudo, são apenas as práticas parentais que predizem os comportamentos de risco e proteção.

\section{Considerações finais}

Esta pesquisa propôs-se a avaliar a influência direta das práticas educativas parentais sobre os comportamentos relacionados à saúde no início da adolescência. Para atingir esse objetivo foram utilizadas técnicas de análise multivariada, uma vez que essas técnicas permitem a avaliação de causa 
e efeito entre variáveis. Os resultados encontrados a partir da análise de regressão múltipla demonstram que as práticas parentais determinam uma parte significativa dos padrões de comportamento competência social, internalização, externalização e problemas de comportamento além de comportamentos de risco e proteção à saúde. Faz-se pertinente destacar que quando apenas as práticas parentais mostram-se como variáveis preditoras de variáveis de estilo pessoal (padrão de comportamento) ou de comportamentos de saúde e risco, fortalece-se a hipótese de que essa fase é importante para o desenvolvimento de hábitos de saúde e que os pais são fundamentais nesse momento. Tais resultados demonstram a relevância do trabalho com pais, principalmente no início da adolescência, sendo esse mais um canal para os trabalhos preventivos.

Como limitações da pesquisa, vale mencionar a forma de coleta de dados a partir de inventários e questionários; esses instrumentos oferecem apenas medidas indiretas do comportamento, ou seja, o que o adolescente relata não necessariamente descreve a forma como ele se comporta em ambiente natural. Pesquisas utilizando metodologias de observação direta e a utilização de múltiplos informantes poderiam contornar essa limitação. Também a ampliação da amostra e a análise de grupos discriminantes possivelmente contribuam para uma melhor validação dos modelos encontrados.

\section{Referências}

Achenbach, T. M. (1991). Manual for the Youth Self-Report and 1991 profile. Burlington: University of Vermont department of psychiatry.

Alvarenga, P. (2001). Práticas educativas parentais como forma de prevenção de problemas de comportamento. In H. J. Guilhardi (Org.). Sobre comportamento e cognição: Expondo a variabilidade (pp. 54-61). Santo André: Esetec.

Barber, B. K., \& Harmon, E. L. (2002). Violating the self: Parental psychological control of children and adolescents. In B. K. Barber (Ed.). Intrusive pareting (pp. 15-52). Wasington: American Psychological Association.

Bee, H. (2003). A criança em desenvolvimento. Porto Alegre: Artmed.
Bronstein, P., Fox, B. J., Kamon, J. L., \& Knolls, M. L. (2007). Parenting and gender as predictors of moral courage in late adolescence: A longitudinal study. Sex Roles, 56(9-10), 661-674.

Camacho, I., \& Matos, M. G. (2007). Práticas parentais educativas, fobia social e rendimento académico em adolescentes. Revista Brasileira de Terapias Cognitivas, 3(3). Recuperado em 16 jan. 2010, de http://pepsic. bvsalud.org/pdf/rbtc/v3n2/v3n2a03.pdf

Carlini, E, A, Galduróz, J. C. F., Noto, A. R., \& Nappo, S. A C. (2002). I Levantamento domiciliar sobre uso de drogas psicotrópicas no Brasil: Estudo envolvendo as 107 maiores cidades do país. São Paulo: Cebrid/Unifesp.

Carlini-Cotrim, B., Gazal-Carvalho, C., \& Gouveia, N. (2000). Comportamentos de saúde entre jovens estudantes das redes pública e privada da área metropolitana do Estado de São Paulo. Revista de Saúde Pública, 34(6), 636-645.

Carmo, J. S., Cunha, L. O., \& Costa, E. C. P. (2009). Adolescência, adolescentes, indivíduos que se comportam: Uma leitura a partir de pressupostos analítico-comportamentais. In. E. R. C. Wielenska (Org.). Sobre comportamento e cognição: Desafios, soluções e questionamentos (pp. 480-487). Santo André: Esetec.

Cecconello, A. M., De Antoni, C., \& Koller, S. H. (2003). Práticas educativas, estilos parentais e abuso físico no contexto familiar. Psicologia em Estudo, 8(n. esp.), 45-54.

Cecconello, A. M., \& Koller, S. H. (2000). Competência social e empatia: Um estudo sobre resiliência com crianças em situação de pobreza. Estudos de Psicologia, 5(1), 71-93.

Cohen, D. A., Richardson, J., \& LaBree, L. (1994). Parenting behaviors and the onset of smoking and alcohol use: A longitudinal study. Pediatrics, 94(3), 368-375.

Conte, F. C. (1997). Promovendo a relação entre pais e filhos. In M. Delitti (Org). Sobre comportamento e cognição (pp. 165-173). Santo André: Artbytes.

Crowley, T. J., Mikulich, S. K., Ehlers, K. M., Shannon, K. B. A., \& Whitmore, E. A. D. (2003). Discriminative validity and clinical utility of an abuse-neglect interview for adolescents with conduct and substance use problems. The American Journal of Psychiatry, 160(8), 1461-1469.

Psicol. Argum. 2013 jul./set., 31 (74), 457-471 
Currie, C., Samdal, O., Boyce, W., \& Smith, R. (2001). Health behavior in school-age children: A World Health Organization cross-national study. Research Protocol for the 2001/02 Survey. Edinburgh: Child and Adolescent Health Research Unit - University of Edinburgh. Recuperado em 21 ago. 2004, de www.hbsc.org.

Dias, S., Matos, M. G., \& Gonçalves, A. (2007). Percepção dos adolescentes acerca da influência dos pais e pares nos seus comportamentos sexuais. Análise Psicológica, 4(25), 625-634.

Dishion, T. J., \& McMahon, R. J. (1998). Parental monitoring and the prevention of child and adolescent problem behavior. A conceptual and empirical formulation. Clinical Child and Family Psychology Review, 1(1), 61-75.

Dodge, K. A., Pettit, G. S., \& Battes, J. E. (1994). Socialization mediators of the relation between socioeconomic status and child conduct problems. Child Development, 65(2), 649-665.

Farias, J. C., Júnior, Nahas, M. V., Barros, M. V. G., Loch, M. R., Oliveira, E. S. A, De Bem M. F. L., \& Lopes, A. S. (2009). Comportamentos de risco à saúde em adolescentes no Sul do Brasil: Prevalência e fatores associados. Revista Panamericana de Salud Publica, 25(4), 344-352.

Fletcher, A. C., Steinberg, L., \& Willians-Wheeler, M. (2004). Parental influences on adolescent problem behavior: Revisiting Stattin and Kerr. Child Development, 75(3), 781-796.

Flouri, E., \& Buchanan, A. (2003). The role of mother involvement and father involvement in adolescent bullying behavior. Journal of Interpersonal Violence, 18, 634-644.

Floyd, R. G., Evans, J. J., \& Mcgrew, K. S. (2003). Relations between measures of Cattell-Horn-Carroll (CHC) cognitive abilities and mathematics achievement across the school-age years. Psychology in the Schools, 40(2), 155-171.

Gomide, P. I. C. (2001). Efeito das práticas educativas no desenvolvimento do comportamento anti-social. In M. L. Marinho \& V. E. Caballo (Org.). Psicologia Clínica e da Saúde (pp. 33-54). Londrina: Eduel.

Gomide, P. I. C. (2003). Estilos Parentais e comportamento anti-social. In A. Del Prette e Z. Del Prette (Org.). Habilidades sociais, desenvolvimento e aprendizagem: Questões conceituais, avaliação e intervenção (pp. 2160). Campinas: Alínea.
Gomide, P. I. C. (2004). Pais presentes, pais ausentes. Petrópolis: Vozes.

Gomide, P. I. C. (2006). Inventário de estilos parentais. Petrópolis: Vozes.

Gomide, P. I. C. (2010). Comportamento moral - Uma proposta para o desenvolvimento das virtudes. Curitiba: Juruá.

Guimarães, A. B. P., Hochgraf, P. B., Brasiliano, S. \& Ingberman, Y. K. (2009). Aspectos familiares de meninas adolescentes dependentes de álcool e drogas. Revista de Psiquiatria Clínica, 36(2):69-74.

Haggerty, R. J., Sherrod, L. R., Gamezy, N., \& Rutter, M. (2000). Stress, risk and resilience in children and adolescents: Process, mechanisms and interventions. New York: Cambridge University Press.

Hair, J. E, Anderson, R. E., Tatham, R. L., \& Black, W. C. (2005). Análise multivariada de dados. Porto Alegre: Bookman.

Hawkins, J. D., Catalano, R., Miller, J. (1992). Risk and protective factors for alcohol and other drug problems in adolescence and early adulthood: Implications for substance abuse prevention. Psychological Bulletin, 112(1), 64-105.

Hoffman, J. P. (1993). Exploring the direct and indirect family effects on adolescent drug use. Journal of Drug Issues, 23(3), 535-557.

Hoffman, J. P. (1994). Investigating the age effects of family structure on adolescent marijuana use. Journal of Youth and Adolescence, 23(2), 215-235.

Hunziker, M. H. L., \& Samelo, M. J. (2011). Controle aversivo. In N. B. Borges \& F. A. Cassas. (Org.). Clínica analítico comportamental: Aspectos teóricos e práticos. Porto Alegre: Artmed.

Kandel, D. B. (1996). The parental and peer contexts of adolescent deviance: An algebra of interpersonal influences. Journal of Drug Issue, 26(2), 289-315.

Kandel, D. B., \& Wu, P. (1995). The contributions of mothers and fathers to the intergenerational transmission of cigarette smoking in adolescence. Journal of Research on Adolescence, 5(2), 225-252.

Kerr, M., \& Stattin, H. (2000).What parents know, how they know it, and several forms of adolescent adjustment: Further supporter for a reinterpretation of monitoring. Developmental Psychology, 36(3), 366-380. 
Kumpfer, K. L., \&Alvarado, R. (2003). Family-strengthening approaches for the prevention of youth problem behavior. American Psychologist, 58(6-7), 457-465.

Laranjeira, R., Pinsky, I., Zaleski, M., \& Caetano, R. (2007). I Levantamento nacional sobre os padrões de consumo de álcool na população brasileira. Brasília: Secretaria Nacional Antidrogas.

Lamborn, S. D., Mounts, N. S., Steinberg, L., \& Dornbusch, S. M. (1991). Patterns of competence and adjustment among adolescents from authoritative, authoritarian, indulgent and neglectful families. Child Development, 62(1), 1049-1065.

Lewinsohn, P. M., Rohde; P., Seeley, J. R., Klein, D. N., \& Gotlib, I. H. (2000). Natural course of adolescent major depressive disorder in a community sample: Predictors of recurrence in young adults. American Journal Psychiatry, 157(10):1584-1591.

Legnani, E., Legnani, R. F. S., Lopes, A. S., Campos, W., Krinski, K., Elsangedy, H. M., Silva, S. G., Krause, M. P. (2009). Comportamentos de risco à saúde em escolares da tríplice fronteira. Revista Brasileira de Atividade Física \& Saúde, 14(1), 28-37.

Löhr, S. S. (2001). Desenvolvimento das habilidades sociais como forma de prevenção. In H. J. Guilhardi, M. B. B. P. Madi, P. P. Queiroz \& M. C. Scoz, (Org.). Sobre comportamento e cognição: Expondo a variabilidade. (Vol. 8, pp. 190-209). Santo André: Esetec.

Maccoby, E. E., \& Martin, J. (1983). Socialization in the context of the family: Parent-child interaction. In P. H. Mussen \& E. M. Hetherington (Org.). Handbook of child psychology: Socialization, personality and social development (pp. 1-101). New York: Wiley.

Malcon, M., Menezes, A., Maia, M., Chatkin, M., \& Victoria, C. (2003). Prevalência e fatores de risco para tabagismo em adolescentes na América do Sul: Uma revisão sistemática da literatura. Revista Panamericana de Salud Publica, 13(4), 222-229.

Martínez, I., \& García, J. F. (2007). Impact of parenting styles on adolescents' self-esteem and internalization of values in Spain. The Spanish Journal of Psychology, 10(2), 338-348.

Masten, A. S., \& Garmezy, N. (1985). Risk, vulnerability and protective factors in the developmental psychopathology. In B. B. Lahey \& A. E. Kazdin (Ed.). Advancesin clinical child psychology. (pp. 1-51). New York: Plenum.
Matthews, K. A., Woodall, K. L., Kenyon, K., \& Jacob, T. (1996). Negative family environment as a predictor of boys' future status on measures of hostile attitudes, interview behavior, and anger expression. Health Psychology, 15(1), 30-37.

Menegatti, C. L. (2002). Interaçoes pais-filhos e depressao infantil: Uma abordagem comportamental. Dissertação de Mestrado em Psicologia da Infância e Adolescência não publicada, Universidade Federal do Paraná, Curitiba.

Mondin, E. M. C. (2008). Práticas educativas parentais e seus efeitos na criação dos filhos. Psicologia Argumento, 26(54), 233-244.

Monteio P., Victora C., \& Barros F. (2004). Fatores de risco sociais, familiares e comportamentais para obesidade em adolescentes. Revista Panamericana de Salud Publica, 16(4), 250-258.

Muris, P., Meesters, C., Morren, M., \& Moorman, L. (2004). Anger and hostility in adolescents: Relationships with self-reported attachment style and perceived parental rearing styles. Journal of Psychosomatic Research, 57(3), 257-264.

Novak, G., \& Peláez, M (2004). Child and adolescence development: A behavioral systems approach. Thousand Oaks: SAGE.

Nurco, D., \& Lerner, M. (1996). Vulnerability to narcotic addiction: Family structure and functioning. Journal of Drug Issues, 26(4), 1007-1095.

Olweus, D. (1980). Familial and temperamental determinants of aggressive behavior in adolescents boys: A causal analysis. Developmental psychology, 16(6), 644-660.

Outeiral, J. O. (2008). Adolescer. (3. ed.). Rio de Janeiro: Revinter.

Oxford, M., Oxford, M. L., Harachi W., Catalano, R. F., \& Abbot, R. D. (2001). Preadolescent predictors of substance initiation: A test of both the direct and mediated effect of family social control factors on deviant peer associations and substance initiation. American Journal of Drug and Alcohol Abuse, 27(4), 599-616.

Paiva, F. S. \& Ronzani, T. M. (2009). Estilos parentais e consumo de drogas entre adolescentes: Revisão sistemática. Psicologia em Estudo, 14(1), 177-183.

Patterson, G. R., Reid, J. B., \& Dishion, T. J. (1992). Antisocial boys. Eugene: Castalia Publishing.

Psicol. Argum. 2013 jul./set., 31 (74), 457-471 
Pettit, G., \& Bates, J. (1989). Family interaction patterns and children's behavior problems from infancy to 4 years. Developmental Psychology, 25(3), 413-420.

Pinto, J. M. M. (2006). Adolescência e grupos: Estrutura, funções, relações e vivências no(s) grupo(s) adolescente(s). Tese de doutoramento em Psicologia Clínica, Faculdade de Psicologia e Ciências da Educação de Coimbra, Coimbra.

Reppold, C. T., Pacheco, J., Bardagi, M., \& Hutz, C. S. (2002). Prevenção de problemas de comportamento e desenvolvimento de competências psicossociais em crianças e adolescentes: Uma análise das práticas educativas e dos estilos parentais. In C. S. Hutz (Org.). Situações de risco e vulnerabilidade na infância e na adolescência: Aspectos teóricos e estratégia de intervenção. (pp.7-51). São Paulo: Casa do Psicólogo.

Sá, D. G. F., Bordin, I. A. S., Martin, D., Paula, C. S. (2010). Fatores de risco para problemas de saúde mental na infância/adolescência. Psicologia: Teoria e Pesquisa, 26(4), 643-652.

Sabbag, G. M. (2003). Validação externa do inventário de estilos parentais: Um estudo de caso com duas famílias de risco. Monografia de conclusão do curso de psicologia, Universidade Federal do Paraná, Curitiba.

Salvador, A. P. V. (2007). Relação entre práticas educativas parentais, envolvimento com tarefas escolares, depressão e desempenho acadêmico de adolescentes. Dissertação de Mestrado em Educação, Universidade Federal do Paraná, Curitiba.

Salvo, C. G. (2010). Práticas educativas parentais e comportamentos de proteção e risco à saúde em adolescentes. Tese de Doutorado em Psicologia Clínica, Universidade de São Paulo, São Paulo.

Salvo, C. G., Silvares, E. F. M., \& Toni, P. M. (2005). Práticas educativas como forma de predição de problemas de comportamento e competência social. Estudos de Psicologia (Campinas), 22(2), 187-196.

Santos, G. E. S. (2001). Intervenção com famílias portadoras de necessidades especiais: O caso de pais agressores. Dissertação de Mestrado em Educação Especial, Universidade Federal de São Carlos, São Carlos.

Sapienza, G., \& Pedromônico, M. R. M. (2005). Risco, proteção e resiliência no desenvolvimento da criança e do adolescente. Psicologia em Estudo, 10(2), 209-216.

Severe, S. (2000). A educação pelo bom exemplo. Rio de Janeiro: Campus.
Shaffer, D. R. (2005). Psicologia do desenvolvimento. São Paulo: Thomson.

Sidman, M. (1995). Coerção e suas implicações. Campinas: Editorial Psy.

Simons, R. L., Wu, C., Lin, K., Gordon, L., \& Conger, R. D. (2000). A cross-cultural examination of the link between corporal punishment and adolescent antisocial behavior. Criminology, 38(1), 47-80.

Stattin, H., \& Kerr, M. (2000). Parental monitoring: A reinterpretation. Child Development, 71(4), 1072-1085.

Stanton, B. F., Xiaoming, L., Galbraith, J., Cornick, G., Feigelman, S., Kaljee, L., \& Zhou, Y. (2000). Parental underestimates of adolescent risk behavior: A randomized, controlled trial of a parental monitoring intervention. Journal of Adolescent Health, 26(1), 18-26.

Steinberg, L., \& Morris, A. S. (2001). Adolescent development. Annual Review of Psychology, 52, 83-110.

Steinberg, L. (2000). The family at adolescence: Transition and transformation. Journal of Adolescent Health, 27(3), 170-178.

Steinberg, L., Lamborn, S. D., Darling, N., Mounts, N. S., \& Dornbusch, S. M. (1994). Over-time changes in adjustment and competence among adolescents from authoritative, authoritarian, indulgent and neglectful families. Child Development, 65(3), 754-770.

Taquette, S. R., Andrade, R. B., Vilhena, M., \& Paula, M. C. (2005). A relação entre as características sociais e comportamentais da adolescente e as doenças sexualmente transmissíveis. Revista da Associação Médica Brasileira, 51(3), 148-152.

Totura, C. M. W., MacKinnon-Lewis, C., Gesten, E. L., Gadd, R., Divine, K. P., Dunham, S. \& Kamboukos, D. (2009). Bullying and victimization among boys and girls in middle school: The influence of perceived family and school contexts. The Journal of Early Adolescence, 29(4), 571-609.

Vuchinich, S., Bank, L., \& Patterson, G. R. (1992). Parenting, peers and the stability of antisocial behavior in preadolescent boys. Developmental Psychology, 28(3), 510-521.

Weber, L. N. D., Viezzer, A. P., \& Brandenburg, O. J. (2003). Estilos parentais e desenvolvimento da criança e do adolescente. Palmadas e surras: Ontem, hoje e amanhã. In M. Z. Brandão \& F. C. S. Conte (Org.). Sobre comportamento e cognição: A história e os avanços, a seleção por conseqüências em ação. (pp. 512- 526). Santo André: Esetec. 
Weber, L. N. D. (2007). Eduque com carinho. Curitiba: Juruá.

Weber, L., \& Gomide, P. I. C. (2004). 0 comportamento moral e os estilos parentais. In M. Z. S. Brandão (Org.). Sobre comportamento e cognição (Vol. 14, pp. 272285). Santo André: Esetec.

Williams, P. G., Holmbeck, G. N., \& Greenley, R. N. (2002). Adolescent health psychology. Journal of Consulting and Clinical Psychology, 70(3), 828-842. 